\title{
Energy Efficient and Congestion Aware Routing Algorithms for Wireless Sensor Networks Connected as Hypercube
}

\author{
Amir Hossein Mohajerzadeh \\ Department of Computer \\ Engineering, Ferdowsi University \\ of Mashad, \\ Mashhad, Iran \\ am_mo84@stu-mail.um.ac.ir
}

\author{
Mohammad Hossien Yaghmaee \\ Department of Computer Engineering, \\ Ferdowsi University of Mashad, \\ Lane Department of Computer Science and \\ Electrical Engineering, West Virginia \\ University, Morgantown, WV 26506 \\ hyaghmae@ferdowsi.um.ac.ir
}

\author{
Zahra Eskandari and Hossein Deldari \\ Department of Computer Engineering, \\ Ferdowsi University of Mashad, \\ Mashhad, Iran \\ za es73@stu-mail.um.ac.ir, \\ hd@ferdowsi.um.ac.ir
}

\begin{abstract}
Wireless Sensor Networks (WSN) have been noticed and researched nowadays. These new type of networks have unique characteristics. WSNs have different limitations such as computational power, storage capacity, energy source and etc. The main constraint of wireless sensor networks is energy. The energy source of sensor nodes is limited and not rechargeable. Therefore, optimizing consumption of energy is essential for WSNs protocols. Hypercube topology is very popular in distributed environments. In last few years, many different topology specific routing algorithms have been designed for wireless sensor networks, but to the best of our knowledge, none of them consider Hypercube as their topology. In this paper, different routing algorithms are proposed for WSN with Hypercube topology. The performance of all proposed routing algorithms is evaluated, and the best algorithm which can provide more fairness is introduced. Simulation results which are represented in last section of this paper are the base of our conclusions. The main goal of this paper is to find a fair energy aware routing algorithm for wireless sensor network connected as Hypercube.
\end{abstract}

Keywords-component; Energy Aware Algorithm; Fairness; Hypercube; Routing Algorithms; Wireless Sensor Networks.

\section{INTRODUCTION}

In recent years, many researches have been done on Wireless Sensor Networks. These networks are very popular nowadays. Sensor network is composed of hundreds or thousands of nodes which have sensors to sense their environment. These networks have unique characteristics which make them different rather than other networks. A sensor node may have different sensors which cooperatively monitor physical or environmental conditions, such as temperature, sound, vibration, pressure, motion or pollutants, at different locations. The sensor nodes collect data from their environment and then send them to one or more main nodes which are called sink. The sink node collects data from other sensor nodes and processes them. Sensor nodes transmit data in ad hoc manner. In ad hoc networks, nodes are responsible to find a best path to forward data to the sink $[1,2]$.

WSNs have many different applications. They are used in commercial, industrial and civilian applications including environment and habitat monitoring, healthcare applications, home automation, traffic control and monitoring, object tracking and fire detection. Each node in a WSN is typically equipped with one or more sensors, a wireless communications device, a processor, and an energy source, usually a battery. Design and deployment of sensor networks are highly depended on their application [5]. In battle field, wireless sensor network topology is dynamic because nodes may drop or move for various reasons. But in environments such as city traffic system and life monitoring systems, network topology is almost static and does not change in its lifetime. With respect to points mentioned before, protocols which are designed for wireless sensor networks with dynamic topology are different from protocols which are designed for static one. The protocols of wireless sensor networks should be designed based on their unique and different characteristics $[8,17]$.

Wireless sensor networks have unique characteristics rather than other networks. Sensor nodes have many constraints such as computational power, storage capacity, communication range, and limited energy source. Of course, the main constraint is energy source. Energy source which is dedicated to sensor nodes is limited and in most of applications, it is not rechargeable. Energy determines the network lifetime. A wireless sensor network can perform its tasks while its nodes have enough energy. Therefore, if energy consumption is reduced the network lifetime will be prolonged. Prolonging network lifetime leads to using network advantages longer. 
Nodes energy is consumed for two main purposes. First, for computations performed in node and second, for node communications which are performed with its neighbors. Communication is the most important factor in node energy consumption. Protocols which are implemented in nodes manage their functions. Because of success of layered model in traditional networks, this model is used for wireless sensor networks too. Each layer uses different protocols to perform its tasks. With respect to points that mentioned before, using energy aware protocols which consider energy in their decision making, can decrease wireless sensor network energy consumption $[3,4]$.

Fairness in energy consumption of sensor nodes has direct affect on the network energy consumption. In WSN all sensor nodes are responsible to forward their local and transient data toward sink node. Therefore, if a node is frequently used by the other nodes for data forwarding, it will lose its energy and will die sooner than the other nodes. The dead nodes lead to wireless sensor network partitioning. When a network is partitioned, its energy consumption will be increased seriously. By using fair routing protocols which provide fairness in energy consumption, the network lifetime will be increased. Routing in wireless sensor networks is different from routing in traditional networks because of their unique characteristics. Different routing algorithms have been designed for wireless sensor networks. Algorithms given in [9, 10] are principal routing algorithms in wireless sensor networks. As mentioned before, energy consumption is the most important factor for routing algorithms of WSNs. Different energy aware routing algorithms have been designed for wireless sensor networks. In $[11,12]$ optimal energy consumption is the most important objective. These algorithms do not consider network topology. As discussed in [9], in different applications if topology is determined, more efficient protocols can be designed. Network dynamics is one of the most important challenges for designing routing protocol for wireless sensor networks. Many applications such as city traffic management, health monitoring systems and security systems, have almost static topology. In these networks by participating topology in designing routing algorithm, more efficient algorithms are achievable. Many researches are conducted on node deployment in wireless sensor networks. This area is still active in wireless sensor networks [13, 14]. In [16, 18] different Grid topology have been proposed for WSNs, but their objectives are different. The main objective of [16] is maximum coverage and that of [18] is minimum energy consumption.

In last few years, many different routing algorithms have been designed for wireless sensor networks, but to the best of our knowledge, none of them considers Hypercube as their topology. In this paper, different routing algorithms for WSN with Hypercube topology are discussed. Using Hypercube has different advantages for a wireless sensor network. Designing a routing protocol for a network with determined topology is more efficient. In static topology, the position of nodes and their relations are determined. It is clear that the Hypercube topology is not suitable for all WSN's applications. In some specific applications such as traffic management, health monitoring, and security we can use Hypercube topology.
These environments have static topology which using Hypercube topology is very efficient [7].

The reminder of this paper is organized as follow. In section 2, Hypercube topology is discussed. In section 3 the similar characteristics of some routing algorithms including Random, Round Robin, Forward Edge, Probabilistic algorithm and algorithms that make decision based on their neighbors' energy are discussed. In section 4 , by using computer simulation the performance of mentioned routing algorithms is evaluated. Finally we conclude the paper in section 5 .

\section{HYPERCUBE TOPOLOGY}

Hypercube topology is popular in distributed systems. In this paper we consider 3-dimensional hypercube. In this topology, the nodes positions are pre determined. All nodes except border one have six neighbors. Border nodes depended on their positions may have 5, 4 and 3 neighbors. A Hypercube topology with eight nodes is depicted in fig.1 [6].

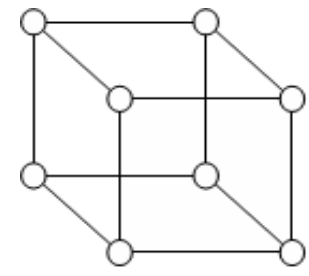

Figure 1. A 3-D Hypercube with eight nodes

Wireless sensor network with hypercube topology is considered as a graph $\mathrm{G}=(\mathrm{V}, \mathrm{E})$, where $\mathrm{V}$ is the set of all sensors and $\mathrm{E}$ is the set of all edges between pairs of sensors. If two nodes are located in their transmission range, an edge is considered between them. As we mentioned earlier, network nodes collect data and forward them to the sink using one of the existing routes. In Hypercube topology, there are many different routes between each source and the sink. Routing algorithm should select one of the mentioned routes to transmit traffic. There are two different Hypercube topologies for wireless sensor networks. First, all the nodes in the Hypercube have a sensor node while in the second topology, many of nodes in Hypercube have a sensor node and others don't have. Second topology is more complicated and less applicable. In this paper the first topology is considered.

In Hypercube topology, each node $(\mathrm{i}, \mathrm{j}, \mathrm{k})$ has at most six neighbors which are: $(\mathrm{i}-1, \mathrm{j}, \mathrm{k}),(\mathrm{i}+1, \mathrm{j}, \mathrm{k}),(\mathrm{i}, \mathrm{j}-1, \mathrm{k}),(\mathrm{i}, \mathrm{j}+1, \mathrm{k}),(\mathrm{i}, \mathrm{j}, \mathrm{k}-$ $1)$ and $(\mathrm{i}, \mathrm{j}, \mathrm{k}+1)$. Border nodes may have 3,4 and 5 neighbors. Neighbors $(\mathrm{i}-1, \mathrm{j}, \mathrm{k}),(\mathrm{i}, \mathrm{j}, \mathrm{k}-1),(\mathrm{i}, \mathrm{j}-1, \mathrm{k})$ have shorter distance to sink rather than the others, and neighbors $(i+1, j, k),(, j+1, k)$, $(\mathrm{i}, \mathrm{j}, \mathrm{k}+1)$ have longer distance. All routing algorithms in wireless sensor networks which are discussed in this paper use neighbors that have shorter distance to the sink. Therefore their energy consumption is minimized. But routing algorithms which do not consider energy constraint may use all the neighbors depended on their objectives. The main goal of this paper is to find a fair energy aware routing algorithm for wireless sensor network connected as Hypercube topology. 


\section{SIMILAR CHARACTERISTICS OF ALL THE ALGORITHMS}

The main task of routing algorithms which are discussed in this paper is to find the best route between source and the sink. All routing algorithm use hop by hop routing strategy. In hop by hop routing strategy, each sensor node only selects its next hop neighbor. In this case, the neighbors which have shorter distance to sink node may be selected as the next hop. Therefore energy consumption of all algorithms is the same. Each algorithm tries to provide fairness as much as possible. To provide fairness in routing process, routing algorithm must select different routes between sender and the sink as much as possible. In the following subsections, we will explain different routing algorithm proposed for WSNs.

\section{A. The Random algorithm}

Random algorithm selects routes randomly. A route between source and sink node is established randomly. As discussed above, routing is performed hop by hop. It means that, when a node receives a packet, depended on its routing algorithm, one of its neighbors is selected as the next hop. This process is continued until the packet is arrived to the sink. To select next hop, Random algorithm uses a random function. Random function selects one of the candidate neighbors as the next hop randomly. Candidate neighbors are the neighbors that have shorter distance to the sink rather than sender node. There is no complicated decision making in random function and selection is done simply.

\section{B. The Round Robin algorithm}

The number of candidate neighbors is depended on the sender position. In Hypercube topology, each node has at least one and at most three candidate neighbors. A node which located in the center of Hypercube has six neighbors; however three of them can be selected as candidate neighbors. The nodes which are located in the same axis by sink have only one candidate neighbor. In Round Robin algorithm, sender node selects its next hop alternatively. In other words, if a node has three candidate neighbors, every three rounds, all candidate nodes will be selected as the next hop once.

\section{The Probabilistic algorithm}

Probabilistic algorithm makes decision based on the number of neighbors. This algorithm selects its next hop from its candidate neighbors based on a selection probability which is calculated using number of candidate neighbors. A node selects a candidate neighbor with higher probability, if it has higher number of candidate neighbors. For example, if a node has three candidate neighbors and another node has only one candidate neighbor, selection probability of the first node will be three times of the second node.

\section{The Edge algorithm}

This algorithm forwards data from source to destination using the edges of Hypercube. Consider a Hypercube that two opposite nodes of its main diameter are source and sink nodes. Edge algorithm uses border nodes for transmitting data toward sink node. This algorithm uses Hypercube topology structure. There are six different routes between each node and the sink which are: $\mathrm{X}-\mathrm{Y}-\mathrm{Z}$ ، $\mathrm{X}-\mathrm{Z}-\mathrm{Y}$ ، $\mathrm{Y}-\mathrm{X}-\mathrm{Z}$ ، $\mathrm{Y}-\mathrm{Z}-\mathrm{X}$ ، Z-X-Y and Z-Y$X$. "X-Y-Z" determines axis that a packet should traverse while it reaches to the sink. Selection between these possible routes is performed alternatively. In other words, in each six rounds each route is selected one time.

\section{E. The VarEdge algorithm}

This algorithm is similar to Edge algorithm. When we simulate algorithm in VarEdge condition, there is multiple sinks with different positions; but in Edge simulation, for all of the experiments there is only one sink with constant position. Sink position is a very important factor for Edge algorithm; therefore we consider these two modes for our experiments.

\section{F. The Algorithms make decision based on their neighbor energy}

Algorithms which were discussed in previous sections do not consider the remaining energy of neighbor nodes. In this section some algorithms that make decision based on the remaining energy of neighbor nodes are discussed. In the first step, the algorithm that only considers the remaining energy of its neighbor is discussed. This algorithm selects neighbor that has highest remaining energy as its next hop. It is observable that, the mentioned algorithm provides fairness using energy as the most important factor. If a node is used more, its energy will be depleted sooner; this node will be used fewer in the future. There is another solution for this challenge. We can use a counter in each node. If a node transmits a packet, its counter will be added by one. By using this method, counter represents the number of packets that a node has transmitted. When a node decides to chose its next hop, it should select a neighbor with lowest value of counter. Lower value of counter means that node has been used less. It is important to note that, the main goal of these algorithms is to provide fairness in consuming nodes energy.

Another algorithm which is discussed here uses two hop neighbors for decision making about its next hop. In this algorithm, when a node wants to determine its next hop, it also considers the energy of its candidate neighbors. Mentioned algorithm considers all of the states for its two hop neighbors. For example, if a node has three candidate neighbors and each of them has two candidate neighbors, there are six different states. For each of these states, the sum of remained energy of first and second hop neighbors is calculated. In fig.2 an example is depicted. Triangle, Square and Diamond nodes are the first hop candidate neighbors of Circle node; each of them has two candidate neighbors. Totally there are six different states for Circle node.

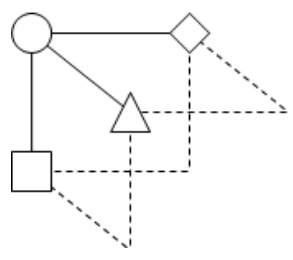

Figure 2. An example of candidate neighbors

Note that collecting information has overhead. In algorithms which are discussed in previous section, nodes do 
not need extra information about their environment except their position in Hypercube topology. But algorithms which are argued in this section need extra information about their first and second hop neighbors. Collecting this extra information has additional overhead.

\section{G. The Congestion Aware algorithm}

In this section, a congestion aware algorithm will be discussed. This algorithm considers its neighbors queue length for decision making. Each network node has a queue for storing arrival packets. In this algorithm, when a packet arrives to a node, candidate neighbor with shortest queue length will be selected for next hop. Using this algorithm packet loss should be decreased, because algorithm selects candidate neighbor with longest free space queue.

\section{Simulation RESUlts}

In this section, routing algorithms which were explained in sections 3 are evaluated. Two different Hypercube topologies are considered which are a 5-5-5 Hypercube, and a 5-15-15 Hypercube. Events are randomly occurred in network boundaries.

We use deviation for comparing fairness of different routing algorithms. Deviation is calculated as below:

$$
\text { Deviation }=(\text { Node'sEnergy }- \text { Average })^{2}
$$

In (1) Average and Node'sEnergy represent the average energy consumption of network nodes and the node's energy respectively. Using (1), deviation is calculated for each network node. We use sum of deviations which are calculated for all network nodes as the evaluation metric. In fig. 3 to evaluate the fairness of each algorithm, the Deviation is plotted versus number of events. The algorithm which has better fairness is more suitable and successful than the others.

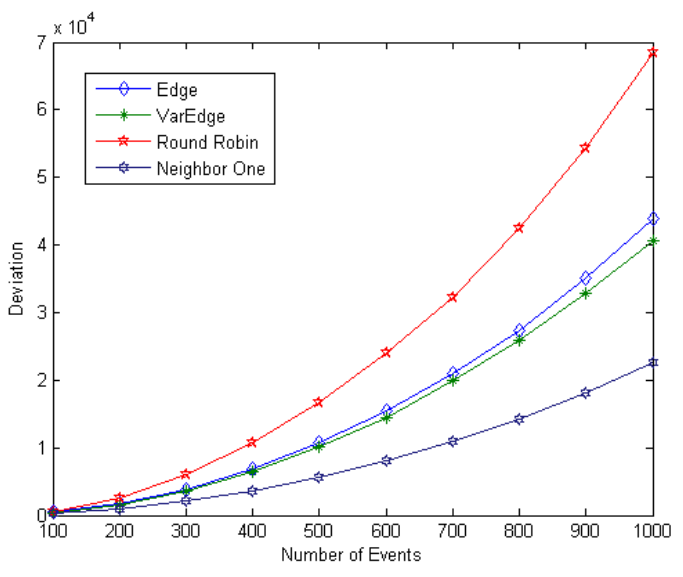

Figure 3. Deviation versus number of events

As it can be seen in fig.3, Neighbor One algorithm has the lowest deviation and so it can provide fairness better than the other algorithms. In fig.4, the performance of Random, Round Robin and Probabilistic algorithms is given.

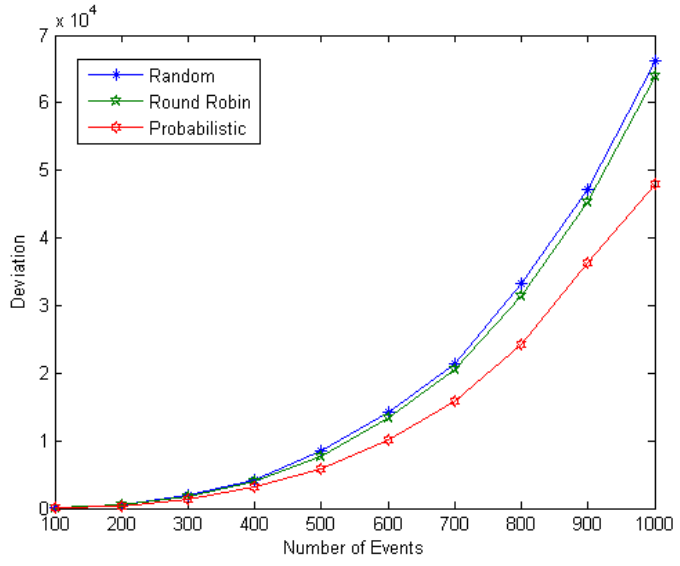

Figure 4. Deviation versus number of events

As it is shown in fig.4, Probabilistic algorithm has lower deviation rather than the other two algorithms. Lower deviation means that reminding energy of network nodes are close together. Total energy consumption of all algorithms which are argued in this paper is equal. In fig.5, for the case that number of events is too high and for different algorithms, Deviation is plotted versus number of events in the network. We evaluated the performance of algorithms in two different situations. The results of fig. 3 and fig. 4 are used to evaluate the performance of algorithms where number of events is less than 1000 while in fig.5 and fig. 6 the number of events is between 1000 and 10000 .

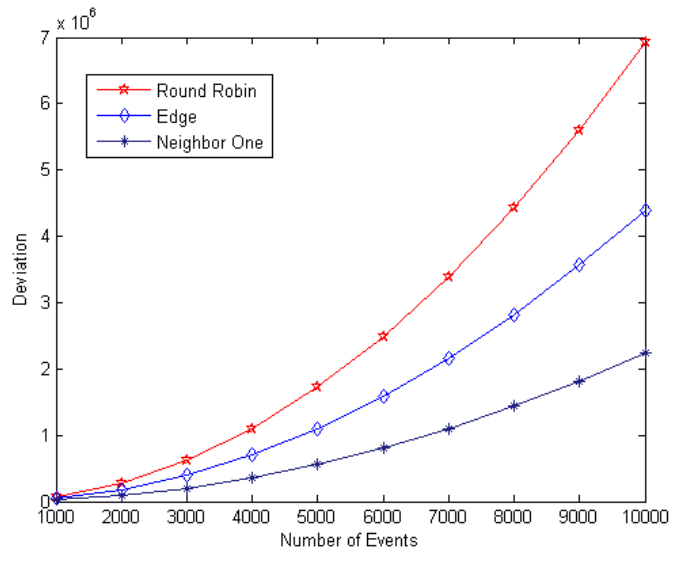

Figure 5. Deviation versus number of events

It is observable that the Neighbor One algorithm has the best performance while the Round Robin algorithm has the worst performance. The VarEdge and Edge algorithms have similar structure; but as discussed earlier, their deployments are different. The VarEdge algorithm is more efficient in network with high number of sinks. In experiments which were performed for Edge algorithm, sink position was considered to be constant but for VarEdge algorithm, six different sinks were considered in different positions. Fig.6 shows simulation results. 


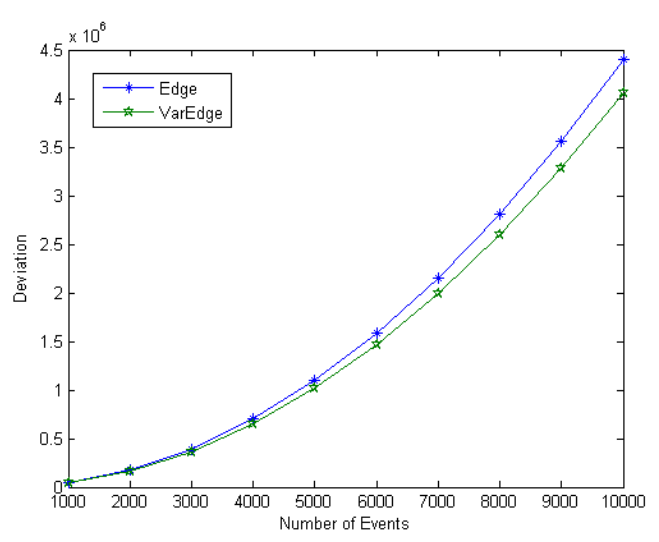

Figure 6. Deviation versus number of events

The other evaluation metric which can help us to compare the performance of different algorithms is packet loss. As mentioned earlier, the nodes in wireless sensor networks die when their energy are depleted. When a node dies, it can not forward packets; therefore packets will be dropped. If all neighbors of a node die, it is not possible to send any packet toward sink. In this case all of sent packets will be dropped. Any lost packet should be retransmitted which consumes more energy. Algorithms which have lower packet loss are more suitable for WSNs. In fig.7 for different routing algorithms, the number of lost packets is plotted versus number of events. It can be seen that the Neighbor One algorithm has the least number of lost packets while the Round Robin algorithm has the most number of lost packets.

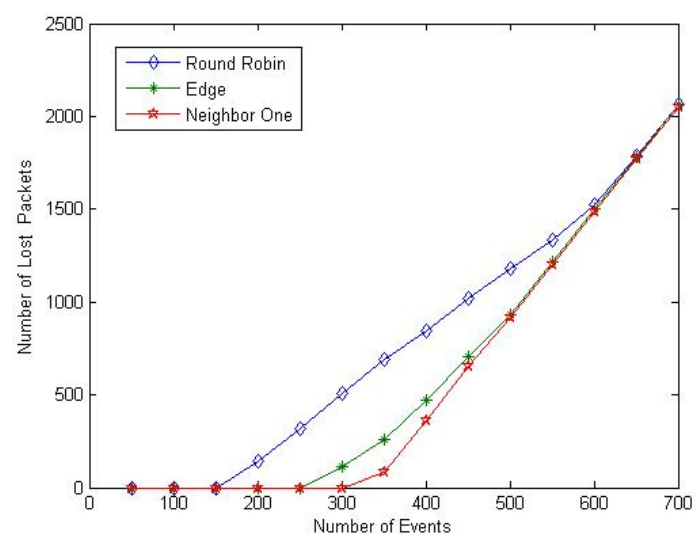

Figure 7. Number of loss packets versus number of events

In fig.8, the packet loss performance of Random, Round Robin and Probabilistic algorithms are compared.

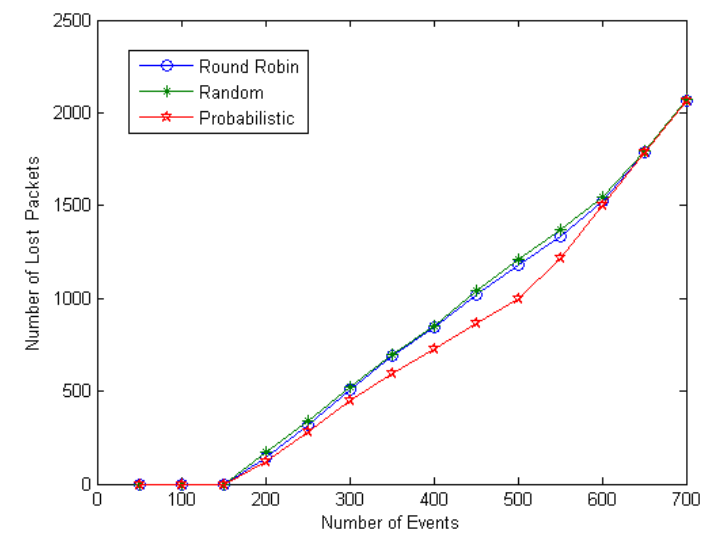

Figure 8. Number of loss packets versus number of events

Congestion aware algorithms are mentioned nowadays specially [15]. In this paper, we proposed a congestion aware routing algorithm in section 3.G. Congestion aware algorithms try to reduce congestion in network nodes. Using nodes queue length, algorithms can avoid congestion occurrence in nodes.

In fig.9 we evaluate proposed congestion aware algorithm performance in comparison with Round Robin. As observable in fig.9, number of loss packets for congestion aware algorithm is less than Round Robin. We can induce that, using congestion aware algorithm make routing more reliable. It is possible to merge congestion aware and energy aware algorithms together. For future work, this type of algorithms can be evaluated.

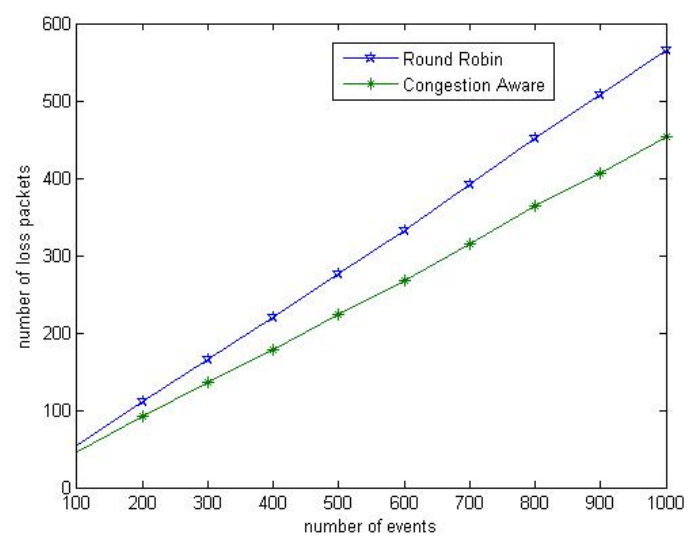

Figure 9. Comparison number of loss packets between congestion aware and Round Robin

\section{CONCLUSION AND FUTURE WORKS}

Wireless Sensor Networks have received significant attention recently due to a wide range of compelling potential applications, such as traffic monitoring, intelligent control systems and digital battle fields. In sensor networks, a large number of small, inexpensive, battery-powered sensors are densely deployed in system environments to capture the status of interest and collect useful information from their surroundings. Fairness in energy consumption of network nodes has direct affect on the network lifetime. In this paper, different routing algorithms of wireless sensor network 
connected as Hypercube are discussed. The main difference of algorithms which were discussed in this paper is their own mechanism to provide fairness in energy consumption. Simulation results declare that which algorithm can prolonged network lifetime more than others. Using other topologies for wireless sensor networks is also applicable. Each of those topologies is efficient for individual application. For future works, evaluating other topologies for wireless sensor networks is suggested.

\section{REFERENCE}

[1] I. F. Akyildiz, W. Su, Y. Sankrasubramaniam, and E. Cayirci, "A survey on sensor networks," IEEE Communication Magazine, August 2002, pp. 102-114.

[2] D. E. Culler and W. Hong, "Wireless sensor networks,"Communications of the ACM, vol. 47, no. 6, June 2004, pp. 30-33.

[3] K. Akkaya and M. Younis, "A Survey on Routing Protocols for Wireless Sensor Networks", Elsevier Ad Hoc Network Journal, Vol. 3/3, 2005, pp. 325-349.

[4] J. N. Al-Karaki and A. E. Kamal, "Routing Techniques in Wireless Sensor Networks: A Survey", IEEECommunications, Vol. 11, No. 6, December 2004, pp.6-28.

[5] Mark Gaynor, Steve Moulton, Matt Welsh, Ed LaCombe, Austin Rowan, John Wynne, "Integrating Wireless Sensor Networks with the Grid", 1089-7801/04, 2006

[6] Yu GE Qinhe YIN Seng Kee TAN Qi YAO Boon Sain YEO Winston SEAH "GREEN: A Grid-based Energy Efficient Probabilistic Routing in Wireless Sensor Networks", 0-7803-8887-9/05, 2005

[7] X. Chen, H. Jia, "Energy Balancing Routing Algorithms in Wireless Sensor Networks Connected as Grids", International Conference on Wireless Algorithms, Systems and Applications, 0-7695-2981-X/07, 2007

[8] Alois Ferscha1, Stephan Olariu, and Tom Pfeifer, "Wireless Sensor Networks and Applications", Dagstuhl Seminar Proceedings 04122, 2006

[9] C. Intanagonwiwat, R. Govindan, D. Estrin, "Directed diffusion: a scalable and robust communication paradigm for sensor networks," Proceeding of ACM MobiCom '00, Boston, MA, 2000, pp.56-67.

[10] T. He, et al, "SPEED: a stateless protocol for real time communication in sensor networks", in the Proceedings of International Conference on Distributed Computing Systems, Providence, RI, May 2003.

[11] Z. Eskandari, M.H. Yaghmaee, A.H. Mohajerzadeh, "Energy Efficient Spanning tree for data aggregation in wireless sensor networks", SN2008 workshop at ICCCN 2008.

[12] Y. H. Wang, C. P. Hsu, Y. C. Lin, C. S. Kuo, H. Y. Ho, "A Routing Method by Reactive Energy Decision in Wireless Sensor Networks", 21 st International Conference on Advanced Information Networking and Applications Workshops (AINAW'07), IEEE 2007

[13] G. Eang, G. Cao, T. La Porta, "Movement assisted sensor depoloyment," Proceeding of IEEE INFOCOM, March 2004.

[14] G. Wang, G. Cao, T. La Porta, W. Zhang, "Sensor relocation in mobile sensor networks," Proceedings of IEEE INFOCOM, 2005.

[15] R. Kumar, "Mitigating Performance Degradation in Congested Sensor Networks", IEEE Transactions on Mobile Computing, Vol.7, No.6, 2008.

[16] K. Chakrabarty, S. S. Iyengar, H. Qi, E. Cho, "Grid coverage for surveillance and target location in distributed sensor networks," IEEE Transaction on Computers, vol. 51, no. 12, 2002, pp. 1448-1453.

[17] A. H. Mohajerzadeh, M. H. Yaghmaee, A. Eskandari, "Tree Based Energy Efficient and Congestion Aware Routing Protocol for Wireless Sensor Networks", $11^{\text {th }}$ IEEE International Conference on Communication Systems, China, 2008, in press

[18] X. Chen, X. Jia, "Energy balancing routing algorithm in wireless sensor networks connected as Grids," International Conference on Wireless Algorithms, Systems and Applications, IEEE 2007. 